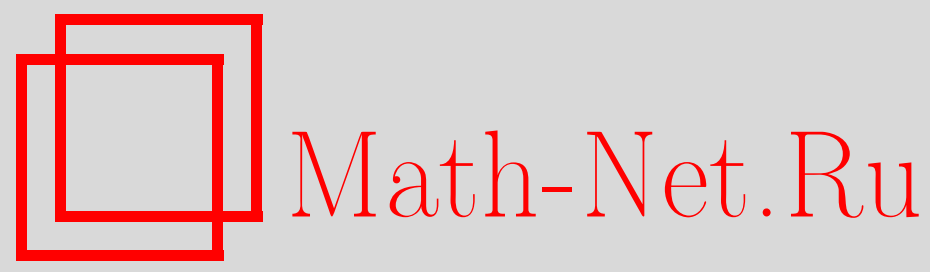

С. А. Назаров, А. С. Слуцкий, Осреднение эллиптической системы при утончении ячеек периодичности в одном направлении, Матем. заметки, 2005, том 78, выпуск 6, 878891

DOI: https://doi.org/10.4213/mzm2660

Использование Общероссийского математического портала Math-Net.Ru подразумевает, что вы прочитали и согласны с пользовательским соглашением http://www . mathnet.ru/rus/agreement

Параметры загрузки:

IP : 18.209 .158 .208

26 апреля 2023 г., 15:30:48

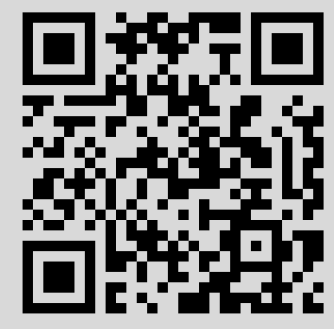




\title{
ОСРЕДНЕНИЕ ЭЛЛИПТИЧЕСКОЙ СИСТЕМЫ ПРИ УТОНЧЕНИИ ЯЧЕЕК ПЕРИОДИЧНОСТИ В ОДНОМ НАПРАВЛЕНИИ
}

\author{
С. А. Назаров, А. С. Слуцкий
}

\begin{abstract}
Произведено осреднение эллиптической системы второго порядка с анизотропным фрактальным строением, характерным для многих реальных объектов: ячейки периодичности утончаются в одном направлении. Рассматривается задача в прямоугольнике, на двух сторонах которого заданы условия Дирихле, а на двух - условия периодичности. Установлена явная формула для осредненного оператора и получена асимптотическая оценка остатка. Точность аппроксимации зависит от показателя $\varkappa \in(0,1 / 2]$ гладкости правой части по медленньм переменным (пространство Соболева-Слободецкого) и оценивается величиной $O\left(h^{\varkappa}\right)$ при $\varkappa \in(0,1 / 2)$ и $O\left(h^{1 / 2}(1+|\log h|)\right)$ при $\varkappa=1 / 2$.
\end{abstract}

Библиографоия: 12 названий.

1. Разветвляющаяся периодичность и задача осреднения. Пусть $q \in \mathbb{N}:=$ $\{1,2, \ldots\}, q>1$ и $S=\left\{(y, z) \in \mathbb{R}^{2}: y \in\left(0, b_{1}\right), z \in\left(0, b_{2}\right)\right\}$ - прямоугольник со сторонами $b_{1}>0$ и $b_{2}>0$. Функцию $\Psi$ назовем периодической $c$-ветвлением в направлении $z$, если при $m=0$ она удовлетворяет условиям

$$
\begin{aligned}
\Psi(0, z) & =\Psi\left(b_{1}, z\right), & & z \in\left(0, b_{2}\right), \\
q^{-m} \Psi(q y, 0) & =\Psi\left(y, b_{2}\right), & & y \in\left(0, b_{1}\right) .
\end{aligned}
$$

Разумеется, в правой части второго соотношения в $(1)$ стоит $b_{1}$-периодическое продолжение $\Psi$ относительно переменной $y$ (в соответствии с требованием первого соотношения в (1)). При $m=0$ и $q=1$ соотношения (1) означают обычную периодичность. Далее всегда считаем, что $q>1$.

Рассмотрим еще один прямоугольник $\Omega=\left(0, a_{1}\right) \times\left(0, a_{2}\right)$ и введем мальй параметр $h>0$ так, чтобы $a_{i}=N_{i} b_{i} h$, причем $N_{i}$ - большие натуральные числа; $i=1,2$. Прямыми $\Gamma_{j}=\left\{x=\left(x_{1}, x_{2}\right): x_{2}=j h b_{2}\right\}$ рассечем $\Omega$ на полоски $\Pi_{j}(h), j=1, \ldots, N_{2}$, имеюшие ширину $h b_{2}$. Каждую из этих полосок разобьем на конгруэнтные прямоугольники $S_{j}^{p}(h) \simeq S_{j}(h)$ с размерами $h q^{-j} b_{1} \times h b_{2}$; при этом $p=1, \ldots, q^{j} N_{1}$. Подчеркнем, что при увеличении номера ряда $j$ основания прямоугольников уменьшаются (см. рис. 1).

Помимо "медленных" переменных $x$ будем использовать "быстрые" переменные $\xi=$ $\left(\xi_{1}, \xi_{2}\right)=h^{-1} x$ и растянутую поперечную координату

$$
\eta^{(j)}=q^{j} \xi_{1}
$$

Работа выполнена при финансовой поддержке Российского фонда фундаментальных исследований, грант № 03-01-00838. 


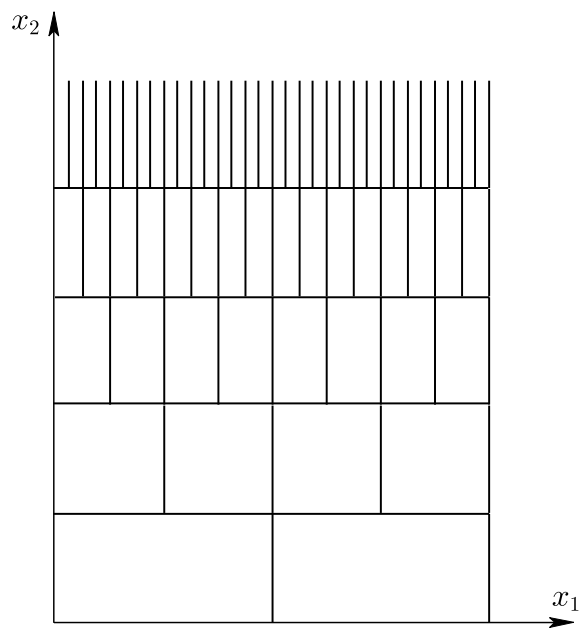

Рис. 1. Случай $q=2, N_{1}=2, h=1$

учитьвающую упомянутое утончение прямоугольника. Далее будет удобно обозначать $\xi_{2}$ также и через $\zeta$, а под $\eta$ понимать переменную, совпадающую с $\eta^{(j)}$ в $\Pi_{j}(h)$, $j=1, \ldots, N_{2}$.

При выполнении соотношений (1) с $m \in \mathbb{N}$ говорим, что у функции $\Psi$ имеется $q$ - $\boldsymbol{в} е т \boldsymbol{\varepsilon}$ ление $c$ коэффициентом $q^{-m}$. Как нетрудно убедиться, при таком свойстве $\Psi$ функция $\Psi^{[m]}$, заданная формулами

$$
\overline{\Pi_{j}(h)} \ni x \mapsto \Psi^{[m]}(\eta, \zeta)=q^{-m j} \Psi(\eta, \zeta), \quad j=1, \ldots, N_{2},
$$

оказьвается непрерьвной в $\bar{\Omega}$. Кроме того, производная $\partial^{m} \Psi / \partial y_{1}^{m}$ становится периодической с $q$-ветвлением, поскольку каждое дифференцирование функции $\Psi$ придает коэффициенту ветвления дополнительньй множитель $q$.

Пусть $D\left(\nabla_{x}\right)$ - матрица размером $k \times n$, составленная из однородных дифференциальных операторов первого порядка с постоянными коэффициентами. Предположим, что $D\left(\nabla_{x}\right)$ удовлетворяет следующему условию алгебраической комплектности [1]: для всякой строки однородных степени $\rho \geqslant \rho_{D}$ полиномов $P=\left(P_{1}, \ldots, P_{n}\right)$ найдется такая строка полиномов $Q=\left(Q_{1}, \ldots, Q_{k}\right)$, что $P\left(t_{1}, t_{2}\right)=Q\left(t_{1}, t_{2}\right) D\left(t_{1}, t_{2}\right)$.

Рассмотрим систему дифференциальных уравнений второго порядка

$$
\mathscr{L}\left(h, x, \nabla_{x}\right) u^{h}(x):=D\left(-\nabla_{x}\right)^{\top} A^{[0]}(x, \eta, \zeta) D\left(\nabla_{x}\right) u^{h}(x)=f^{h}(x), \quad x \in \Omega,
$$

где $u^{h}=\left(u_{1}^{h}, \ldots, u_{n}^{h}\right)^{\top}, f^{h}=\left(f_{1}^{h}, \ldots, f_{n}^{h}\right)^{\top}, \top-$ знак транспонирования, $A$ - матрица размером $k \times k$, ее элементами служат гладкие вещественные функции переменных $x \in \bar{\Omega}, y \in\left[0, b_{1}\right], z \in\left[0, b_{2}\right]$, которые вместе со своими производными по переменным $x$ и $z$ являются $a_{1}$-периодическими относительно $x$ и периодическими относительно $(y, z)$ с $q$-ветвлением в направлении $z$, а производные $\nabla_{x}^{\alpha} \partial_{y}^{m} \partial_{z}^{p} A(x, y, z)$ обладают $q$-ветвлением с коэффициентом $q^{-m}$. Если зависимость от $\eta$ и $\zeta$ определена согласно (3), матрица $A^{[0]}(x, \eta, \zeta)$ гладкая в $\bar{\Omega}$.

Рис. 2 иллюстрирует частньй случай разветвляющейся периодичности, а именно, на нем показано, как "размножается" возмущение (затемненные пятна) постоянной матрицы коэффициентов $A$ согласно правилу (3) при $q=3$. Далее в п. 4 (формула (38) $)$ предполагается аналогичная структура правой части $f^{h}$ : гладкая функция может быть возмущена, например, на тех же затемненных участках области $\Omega$. Подчеркнем, что пятна 


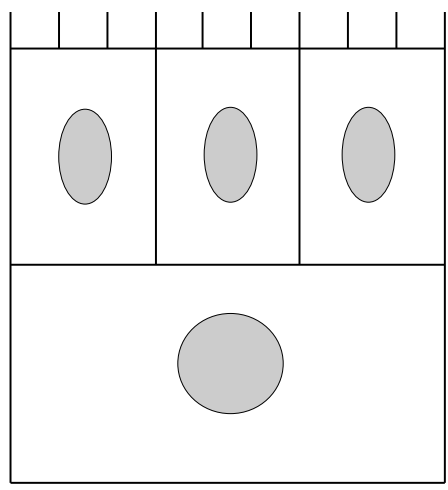

Рис. 2. Случай $q=3, N_{1}=1, h=1$

не являются подобными - их форма меняется в соответствии с размерами содержащих их ячеек $S_{j}^{p}$.

На сторонах прямоугольника $\Omega$ поставим условия Дирихле и условия периодичности

$$
\begin{gathered}
u^{h}\left(x_{1}, 0\right)=0, \quad u^{h}\left(x_{1}, a_{2}\right)=0, \quad x_{1} \in\left(0, a_{1}\right), \\
u^{h}\left(0, x_{2}\right)=u^{h}\left(a_{1}, x_{2}\right), \quad \partial_{x_{1}} u^{h}\left(0, x_{2}\right)=\partial_{x_{1}} u^{h}\left(a_{1}, x_{2}\right), \quad x_{2} \in\left(0, a_{2}\right) .
\end{gathered}
$$

Матрицу $A$ считаем симметрической и положительно определенной при всех $(\eta, \zeta) \in \overline{S_{j}}$. В этом случае благодаря алгебраической комплектности оператор $\mathscr{L}$ оказьвается $ф о р$ мально положительным [1] и обладает полиномиальным свойством [2], [3].

Введем подпространство $H_{\mathrm{per}}^{1}(\Omega)$ функций из $H^{1}(\Omega)$, удовлетворяющих условиям $\left(5_{1}\right)$ и $\left(5_{2}\right)$, и обозначим $H_{\mathrm{per}}^{-1}(\Omega)=\stackrel{\circ}{H}_{\mathrm{per}}^{1}(\Omega)^{*}$ сопряженное пространство. Следующее утверждение известно (см. [1], [2]) и основьвается на неравенстве Корна

$$
\left\|u^{h} ; H^{1}(\Omega)\right\| \leqslant c\left\|D u^{h} ; L_{2}(\Omega)\right\|,
$$

обеспеченном алгебраической комплектностью и условием Дирихле $\left(5_{1}\right)$.

ЛЕмма 1. При введенных выше предположсениях задача (4), (5) является әллиптической, отображсение $\mathscr{L}: \stackrel{\circ}{H}_{\mathrm{per}}^{1}(\Omega)^{n} \rightarrow H_{\mathrm{per}}^{-1}(\Omega)^{n}-$ изоморфизмом, а для (обобщенного) решения задачи (4), (5) $u^{h} \in \stackrel{\circ}{H}_{\mathrm{per}}^{1}(\Omega)^{n}$ выполняется неравенство

$$
\left\|u^{h} ; H^{1}(\Omega)\right\| \leqslant c\left\|f^{h} ; H_{\mathrm{per}}^{-1}(\Omega)\right\|,
$$

в котором постоянная с не зависит от параметра $h \in\left(0, h_{0}\right]$.

Основная цель статьи - осреднение задачи (4), (5) и построение асимптотики ее решения при $h \rightarrow+0$. Зависимость матрицы коэффициентов $A$ от малого параметра $h$ характеризуется измельчением ячеек периодичности в одном направлении и соответствует геометрическим структурам, свойственньм кровеносным, вегетативным или мелиорационньп системам. Подобное строение могут иметь и упругие тела, изготовленные искусственно (специальные каркасные конструкции) или подвергшихся определенным воздействиям (так называемые трещины серебра - craze). Если бы ширина полоски $\Pi_{j}(h)$ составляла $h q^{-j} b_{2}$, и, следовательно, ячейки $S_{j}^{p}(h)$ имели размеры $h q^{-j} b_{1} \times$ 
$h q^{-j} b_{2}$ и оказались подобньми, то возникла бы система дифференщиальных уравнений фрактального типа в тонком прямоугольнике со сторонами $a_{1}$ и $h b_{2}\left(1-q^{-1}\right)^{-1}$ (последний сомножитель - сумма геометрической прогрессии со знаменателем $1 / q)$, причем сопутствующая система уравнений на ячейке периодичности, приобретающей размеры $b_{1} \times b_{2}\left(1-q^{-1}\right)^{-1}$, сохранила бы фрактальньй тип (ср. с [4], [5] и др.).

В рассматриваемой ситуации измельчение производится лиш в одном направлении и высота $h b_{2}$ ячеек остается неизменной. Это обстоятельство, с одной стороны, вынуждает модифицировать стандартный в теории осреднения асимптотический анзац (см. [6]-[8] и др.), усложнив его за счет привлечения анзацев, обслуживающих тонкие области (см. [9], [10] и др.), но с другой стороны, позволяет получить явные выражсения (28), (25) для матрищы А в осредненной задаче

$$
\begin{gathered}
D\left(-\nabla_{x}\right)^{\top} \mathbf{A}(x) D\left(\nabla_{x}\right) v(x)=\mathbf{f}(x), \quad x \in \Omega, \\
v\left(x_{1}, 0\right)=0, \quad v\left(x_{1}, a_{2}\right)=0, \quad x_{1} \in\left(0, a_{1}\right), \\
v\left(0, x_{2}\right)=v\left(a_{1}, x_{2}\right), \quad \partial_{x_{1}} v\left(0, x_{2}\right)=\partial_{x_{1}} v\left(a_{1}, x_{2}\right), \quad x_{2} \in\left(0, a_{2}\right) .
\end{gathered}
$$

Эти формулы громоздки, но используют лишь интегрирование и алгебраические операции.

В части обоснования полученных асимптотических формул следует обратить внимание на несколько новых моментов. Во-первых, зависимость правой части $f=F^{[0]}$ от медленной переменной $x$ соответствует классу Соболева-Слободецкого $H^{\varkappa}$ с дробным показателем $\varkappa \in(0,1 / 2]$, а лемма 8 мажорирует асимптотический остаток величиной $O\left(h^{\varkappa}\right)$. Во-вторых, в лемме 8 фигурируют $L_{\infty}$-нормы функций быстрых переменных и поэтому конструируются три члена асимптотики решения вспомогательной задачи на тонкой области $S_{j}(1)$, удовлетворяющие уравнениям поточечно с погрешностью $O\left(q^{-j}\right)$. В-третьих, относительная толщина ячейки равна $O\left(q^{-j}\right)$ и является малым параметром только при больших номерах $j$, а значит, главньй член невязки, оставленной глобальным асимптотическим приближением в системе (4), составляет $O\left(h^{-1} q^{-j}\right)$ и неограниченно возрастает при $h \rightarrow+0$ и фиксированном $j$. Оценить по норме пространства $H^{1}(\Omega)$ разность истинного решения $u$ и решения $v$ осредненной задачи (7), подправленного асимптотическим корректором, удается благодаря различным вариантам неравенства Харди и соотношению $q^{-j}=O\left(\exp \left(-h \delta x_{2}\right)\right)$, в котором $\delta=b_{2}^{-1} \log q>0$, а точка $x$ берется из полоски $\Pi_{j}(h)$.

2. Предварительные (но безуспешные) преобразования. Сначала произведем формальное осреднение в каждой из полос $\Pi_{j}(h)$, для чего попытаемся воспользоваться обычным анзацем без учета $q$-ветвления:

$$
v(x)+h V^{j}(x, \xi) D\left(\nabla_{x}\right) v(x)+\cdots
$$

Укажем правило дифференцирования сложной функции

$$
D\left(\nabla_{x}\right) \Psi(x, \xi)=D_{x} \Psi(x, \xi)+h^{-1} D_{\xi} \Psi(x, \xi) .
$$

Здесь оператор $D_{x}:=D\left(\nabla_{x}\right)$ дифференцирует по первому аргументу, а $D_{\xi}:=D\left(\nabla_{\xi}\right)-$ по второму. Подставив анзац (8) в систему (4) и собрав члены порядка $h^{-1}$, приходим к уравнению

$$
-D_{\xi}^{\top} A D_{\xi} V^{j}=D_{\xi}^{\top} A \quad \text { на } \quad S_{j}(1),
$$


которое дополним условиями периодичности с $q$-ветвлением

$$
\begin{gathered}
V^{j}\left(x, q \xi_{1}, 0\right)=V^{j}\left(x, \xi_{1}, b_{2}\right), \quad \partial_{\xi_{2}} V^{j}\left(x, q \xi_{1}, 0\right)=\partial_{\xi_{2}} V^{j}\left(x, \xi_{1}, b_{2}\right), \quad \xi_{1} \in\left(0, b_{1} q^{-j}\right) \\
V^{j}\left(x, 0, \xi_{2}\right)=V^{j}\left(x, b_{1} q^{-j}, \xi_{2}\right), \quad \partial_{\xi_{1}} V^{j}\left(x, 0, \xi_{2}\right)=\partial_{\xi_{1}} V^{j}\left(x, b_{1} q^{-j}, \xi_{2}\right), \quad \xi_{2} \in\left(0, b_{2}\right) .
\end{gathered}
$$

Обозначим $\mathscr{H}^{1}\left(S_{j}(1)\right)$ подпространство в пространстве Соболева $H^{1}\left(S_{j}(1)\right)$, состоящее из функций, которые удовлетворяют первым условиям из $\left(11_{1}\right)$ и $\left(11_{2}\right)$. Нетрудно убедиться в том, что при $\mathfrak{F} \in L_{2}\left(S_{j}(1)\right)^{m}$ уравнение

$$
-D_{\xi}^{\top} A D_{\xi} \mathfrak{X}=\mathfrak{F} \quad \text { на } S_{j}(1)
$$

с условиями периодичности вида $(11)$ имеет решение $\mathfrak{X} \in \mathscr{H}\left(S_{j}(1)\right)^{m}$ в том и только том случае, если

$$
\int_{S_{j}(1)} \mathfrak{F}(\xi) d \xi=0 .
$$

Интегрируя по частям при учете периодичности с $q$-ветвлением матрицы $A$, убеждаемся в справедливости условия (12) для $\mathfrak{F}=D_{\xi}^{\top} A$. Таким образом, решение задачи $(10),(11)$ существует.

Соберем коэффициенты при $h^{0}$ в уравнении (4), переписанном при учете формул (8), (9), и получим

$$
-D_{\xi}^{\top} A D_{\xi} v_{2}^{j}=D_{x}^{\top} A D_{x} v+D_{\xi}^{\top} A D_{x} V^{j} D_{x} v+D_{x}^{\top} A D_{\xi} V^{j} D_{x} v+F \quad \text { на } S_{j}(1) .
$$

Условие (12) разрешимости уравнения (13) с условиями периодичности (11) имеет вид

$$
\mathbf{L}^{j}\left(x, \nabla_{x}\right) v=\mathbf{F}^{j}(x)
$$

где $\mathbf{L}^{j}\left(x, \nabla_{x}\right)=-D_{x}^{\top} \mathbf{A}^{j}(x) D_{x}$ и

$$
\begin{aligned}
& \mathbf{A}^{j}(x)=\left|S_{j}(1)\right|^{-1} \int_{S_{j}(1)}\left\{A(x, \xi)+A(x, \xi) D_{\xi} V^{j}(x, \xi)\right\} d \xi \\
& \mathbf{F}^{j}(x)=\left|S_{j}(1)\right|^{-1} \int_{S_{j}(1)} F\left(x, \xi_{1}, q^{j} \xi_{2}\right) d \xi .
\end{aligned}
$$

3. Осреднение задачи. Поскольку данные $\mathbf{L}^{j}$ и $\mathbf{F}^{j}$ зависят от номера $j$ полоски $\Pi_{j}(h) \ni x$, уравнение (14) не поддается осмыслению. Тем не менее, в дальнейшем мы убедимся, что предел $\mathbf{L}^{j}$ при $j \rightarrow+\infty$ как раз и является правильным осредненньм оператором. Дело в том, что относительная толщина ячейки $S_{j}(1)$ составляет $O\left(q^{-j}\right)$ и оказьвается малой при большом $j$. Поэтому для самого корректора $V^{j}$ разумно применить асимптотический анзац, приемлемый для решений краевых задач в тонких областях и обеспеченньй полиномиальным свойством системы (13) (см. [11], [2]). Положим

$$
V^{j}(\varepsilon, x, \xi)=w(x, \zeta)+q^{-j} W\left(x, \eta^{(j)}, \zeta\right)+q^{-2 j} U\left(x, \eta^{(j)}, \zeta\right)+\cdots
$$

где $\eta^{(j)}$ и $\zeta-$ быстрые переменные (см. (2)). Далее по возможности не указьваем индекс $j$. 
Матрица $D_{\xi}$, записанная при помощи переменных $(\eta, \zeta)$, представляется как сумма

$$
\begin{gathered}
D_{\xi}=D\left(\partial_{\xi_{1}}, \partial_{\xi_{2}}\right)=q^{j} D_{\eta}+D_{\zeta} \\
D_{\eta}=D\left(\partial_{\eta}, 0\right)=D_{1} \partial_{\eta}, \quad D_{\zeta}=D\left(0, \partial_{\zeta}\right)=D_{2} \partial_{\zeta}, \quad D_{i}=D\left(\delta_{1, i}, \delta_{2, i}\right) .
\end{gathered}
$$

Подставим формулы (16) и (17) в систему (10) и соберем коэффициенты при одинаковых степенях $q^{-j}$. Множитель при $q^{j}$ имеет вид $D_{\eta}^{\top} A D_{\eta} W+D_{\eta}^{\top} A+D_{\eta}^{\top} A D_{\zeta} w$ и, следовательно, появляется такая система обыкновенных дифференциальных уравнений на отрезке $\left(0, b_{1}\right) \ni y$ с параметрами $x \in \Omega$ и $z \in\left(0, b_{2}\right)$ :

$$
-D_{y}^{\top} A(x, y, z) D_{y} W(x, y, z)=D_{y}^{\top} A(x, y, z)+D_{y}^{\top} A(x, y, z) D_{z} w(x, z)
$$

здесь $D_{y}=D_{1} \partial_{y}, D_{z}=D_{2} \partial_{z}$. Из соотношений (11) вытекает обычное условие периодичности по переменной $y$

$$
W(x, 0, z)=W\left(x, b_{1}, z\right), \quad D_{1}^{\top} A(x, 0, z) D_{y} W(x, 0, z)=D_{1}^{\top} A\left(x, b_{1}, z\right) D_{y} W\left(x, b_{1}, z\right) .
$$

Поскольку $D_{y}^{\top} A D_{y}=\partial_{y} D_{1}^{\top} A D_{y}$, интегрируя по $y$ при учете второго равенства в $(18)$, находим, что

$$
-D_{1}^{\top} A(x, y, z) D_{1} \partial_{y} W(x, y, z)=D_{1}^{\top} A(x, y, z)+D_{1}^{\top} A(x, y, z) D_{z} w(x, z)-C(x, z)
$$

где $C(x, z) \in \mathbb{R}^{k \times n}$ при всех $x$ и $z$.

ПРЕДЛОЖЕНИЕ 2. Матрица $D_{1}^{\top} A D_{1}$ размером $n \times n$ симметрическая и положительно определенная.

ДокАЗАтЕльство. Ясно, что упомянутая матрица симметрична. Кроме того, благодаря предположению о матрице $A$ имеем $\mathscr{X}^{\top} D_{1}^{\top} A D_{1} \mathscr{X} \geqslant c_{A}\left|D_{1} \mathscr{X}\right|^{2}$ при любом столбце $\mathscr{X} \in \mathbb{R}^{n}$; здесь $c_{A}>0$. Осталось убедиться в том, что $D_{1} \mathscr{X} \in \mathbb{R}^{n} \backslash\{0\}$, если только $\mathscr{X} \neq 0$. Для любого столбца $\mathscr{X} \in\left(\mathbb{R}^{n}\right)^{\top}$ свойство алгебраической комплектности матрицы $D$ доставляет такую полиномиальную строку $Q$, что

$$
Q\left(t_{1}, t_{2}\right) D\left(t_{1}, t_{2}\right)=P\left(t_{1}\right):=\mathscr{X} t_{1}^{\rho_{D}} \text {. }
$$

Положим $t_{2}=0$. Заметим, что в силу $(20) Q\left(t_{1}, 0\right)=\mathscr{Y} t_{1}^{\rho_{D}}$ при некоторой строке $\mathscr{Y} \in\left(\mathbb{R}^{m}\right)^{\top}$, и преобразуем (20) в формулу $\mathscr{Y} D_{1}=\mathscr{X}^{\top}$. Следовательно, $\mathscr{Y} D_{1} \mathscr{X}=|\mathscr{X}|^{2}$, т.е. при $\mathscr{X} \neq 0$ равенство $D_{1} \mathscr{X}=0$ в самом деле невозможно.

Теперь соотношение (19) переписьвается следующим образом:

$$
D_{y} W=-\left\{D_{1}\left[D_{1}^{\top} A D_{1}\right]^{-1} D_{1}^{\top} A+D_{1}\left[D_{1}^{\top} A D_{1}\right]^{-1} D_{1}^{\top} A D_{z} w\right\}+D_{1}\left[D_{1}^{\top} A D_{1}\right]^{-1} C .
$$

Так как матрица-функция $W$ периодична относительно переменной $y$, выражение $(21)$ обязано иметь нулевое среднее по $y \in\left(0, b_{1}\right)$, а значит, матрица $C(x, z)$ определяется так:

$$
C=\left\langle\left[D_{1}^{\top} A D_{1}\right]^{-1}\right\rangle_{1}^{-1}\left(\left\langle\left[D_{1}^{\top} A D_{1}\right]^{-1} D_{1}^{\top} A\right\rangle_{1}+\left\langle\left[D_{1}^{\top} A D_{1}\right]^{-1} D_{1}^{\top} A\right\rangle_{1} D_{z} w\right)
$$

Здесь и далее

$$
\langle\Phi\rangle_{i}(x)=\frac{1}{b_{i}} \int_{0}^{b_{i}} \Phi(x, y) d y_{i}, \quad i=1,2 .
$$


Уничтожая коэффициент при $q^{0}$ в уравнении (10), преобразованном согласно (16), получаем для матрицы-функции $U$ систему

$$
-D_{y}^{\top} A D_{y} U=G:=D_{z}^{\top} A+D_{z}^{\top} A D_{y} W+D_{y}^{\top} A D_{z} W+D_{z}^{\top} A D_{z} w,
$$

дополненную условиями периодичности вида (18). Полученная задача разрешима при выполнении условия ортогональности $\langle G\rangle_{1}=0$ (см. (22)), которое благодаря формуле (21) становится уравнением для неизвестной $w$ :

$$
-D_{z}^{\top} A^{1}(x, z) D_{z} w(x, z)=D_{z}^{\top} A^{1}(x, z), \quad z \in\left(0, b_{2}\right) .
$$

При этом $(k \times k)$-матрица $A^{1}=\mathscr{A}(A ; 1)$ вьглядит следующим образом:

$$
\begin{aligned}
\mathscr{A}(A ; i)= & \langle A\rangle_{i}-\left\langle A D_{i}\left[D_{i}^{\top} A D_{i}\right]^{-1} D_{i}^{\top} A\right\rangle_{i} \\
& +\left\langle A D_{i}\left[D_{i}^{\top} A D_{i}\right]^{-1}\right\rangle_{i}\left\langle\left[D_{i}^{\top} A D_{i}\right]^{-1}\right\rangle_{i}^{-1}\left\langle\left[D_{i}^{\top} A D_{i}\right]^{-1} D_{i}^{\top} A\right\rangle_{i} .
\end{aligned}
$$

Лемма 3. Матрица $A^{1}$ симметрическая и положительно определенная.

ДокАЗАТЕЛьСтво. Симметричность не вызьвает сомнений. В силу (21)

$$
\begin{gathered}
D_{y} W=\mathscr{W} D_{z} w+\mathscr{W}, \\
\mathscr{W}=-D_{1}\left[D_{1}^{\top} A D_{1}\right]^{-1} D_{1}^{\top} A+D_{1}\left[D_{1}^{\top} A D_{1}\right]^{-1}\left\langle\left[D_{1}^{\top} A D_{1}\right]^{-1}\right\rangle_{1}^{-1}\left\langle\left[D_{1}^{\top} A D_{1}\right]^{-1} D_{1}^{\top} A\right\rangle_{1} .
\end{gathered}
$$

Прямыми алгебраическими преобразованиями получаем соотношение

$$
A^{1}=\left\langle A+\mathscr{W}^{\top} A+A \mathscr{W}+\mathscr{W}^{\top} A \mathscr{W}\right\rangle_{1}=\left\langle(\mathbf{I}+\mathscr{W})^{\top} A(\mathbf{I}+\mathscr{W})\right\rangle_{1},
$$

где $\mathbf{I}$ - единичная $(k \times k)$-матрица. Таким образом, равенство $\mathscr{X}^{\top} A^{1} \mathscr{X}=0$ возможно лишь при условии $(\mathbf{I}+\mathscr{W}(x, y, z)) \mathscr{X}=0$. Однако в силу вытекающего из (26) соотношения $\langle\mathscr{W}\rangle_{1}=0$ оно вьполнено только в случае $\mathscr{X}=0$.

Для функции $w$, не зависящей от переменной $y$, условие периодичности с $q$-ветвлением в направлении $\zeta$ означает обычную периодичность

$$
w(x, 0)=w\left(x, b_{2}\right), \quad D_{2}^{\top} A^{1}(x, 0) D_{z} w(x, 0)=D_{2}^{\top} A^{1}\left(x, b_{2}\right) D_{z} w\left(x, b_{2}\right) .
$$

Решая задачу (24), (27) по прежней схеме, находим, что

$$
\begin{aligned}
D_{z} w & =-D_{2}\left[D_{2}^{\top} A^{1} D_{2}\right]^{-1} D_{2}^{\top} A^{1}+D_{2}\left[D_{2}^{\top} A^{1} D_{2}\right]^{-1} \mathbf{C}, \\
\mathbf{C} & =\left\langle\left[D_{2}^{\top} A^{1} D_{2}\right]^{-1}\right\rangle_{2}^{-1}\left\langle\left[D_{2}^{\top} A^{1} D_{2}\right]^{-1} D_{2}^{\top} A^{1}\right\rangle_{2} .
\end{aligned}
$$

Таким образом, построен (формально, но здесь обоснование и не требуется) главньй член $\mathbf{V}=V^{\infty}$, асимптотики конгломерата $V^{j}$ из (16), а значит, и главньй член $\mathbf{A}$ матрицы $\left(15_{2}\right)$. Поскольку $D_{\xi}\left(w+q^{-j} W\right)=D_{z} w+D_{y} W+q^{-j} D_{z} W$, имеем

$$
\mathbf{A}=\mathscr{A}\left(A^{1} ; 2\right) \text {. }
$$

Именно эта матрища фигурирует в осредненной задаче (7) с матричным оператором

$$
\mathbf{L}\left(x, \nabla_{x}\right)=D\left(-\nabla_{x}\right)^{\top} \mathbf{A}(x) D\left(\nabla_{x}\right) .
$$

Формулы (28) и (25) дают явное выражение для матрицы $\mathbf{A}$, содержащее лишь интегрирование и алгебраические преобразования. Подчеркнем, что вычисление А производится повторением одной и той же операции: сначала в соответствии с (25) производится осреднение по переменной $y$, а потом - по переменной $z$. 
ПРЕДЛОЖЕНИЕ 4. Матрица А симметрическая и положительно определенная.

ДокАЗАТЕЛЬСТво. Нужно лишь заметить, что формула (28) получается из (25) при замене $A, D_{1}, b_{1}, y$ на $A^{1}, D_{2}, b_{2}, z$ соответственно.

Из эллиптичности оператора $\mathbf{L}$, обеспеченной предложением 4 и алгебраической комплектностью матрицы $D$, в согласии с [12] вытекает очередное утверждение (см. [2], [3]).

Лемма 5. Если $\mathbf{f} \in H_{\mathrm{per}}^{\varkappa}(\Omega)^{n} u \varkappa \in(0,1 / 2]$, то существует единственное решение $v$ задачи (7) из пространства Соболева-Слободецкого $H_{\mathrm{per}}^{2+\varkappa}(\Omega)^{n}$ и верна оценка

$$
\left\|v ; H^{2+\varkappa}(\Omega)\right\| \leqslant c\left\|\mathbf{f} ; H^{\varkappa}(\Omega)\right\| .
$$

Докажем, что несмотря на наличие в правой части (16) множителей $q^{-j}$, члены асимптотики матрицы-функции $V^{j}$ не претерпевают скачков на линиях $\Gamma_{j}(h)$.

Лемма 6. Пусть функиия $Y$ является периодической по переменной у с нулевым средним и обладает q-ветвлением с коэффициентом $q^{-m}$, а функиия $B$ является периодической с q-ветвлением в направлении z. Тогда решение уравнения

$$
\partial_{y} Z(y, z)=Y(y, z), \quad y \in\left(0, b_{1}\right), \quad z \in\left(0, b_{2}\right)
$$

подчинено условию $\int_{0}^{b_{1}} Z(y, z) d y=0$ при $z \in\left(0, b_{2}\right)$ и произведения $B Z, B Y$ обладают $q$-ветвлением с коэффиииентом $q^{-m-1}$.

ДокАЗАТЕЛьство. В силу (30) справедлива цепочка равенств

$$
\begin{aligned}
Z\left(y, b_{2}\right) & =\int_{0}^{y} Y\left(t, b_{2}\right) d t=q^{-m} \int_{0}^{y} Y(q t, 0) d t \\
& =q^{-m-1} \int_{0}^{q y} Y(T, 0) d T=q^{-m-1} Z(q y, 0) .
\end{aligned}
$$

Таким образом, нужное свойство функции $Z$ установлено. Утверждение о произведениях очевидно.

СледСТВИЕ 7. Матрицы-функиии $W$ и $U$ обладают q-ветвлением с коэффиииентами $q^{-1}$ и $q^{-2}$ соответственно.

ДокАЗАТЕЛЬСТво. Слагаемые в правой части (19) являются произведениями периодических с $q$-ветвлением функций, и лемма 6 устанавливает $q$-ветвление с коэффициентом $q^{-1}$ у решения $W$. Многократньм применением леммы 6 к слагаемьм в равенстве (23) проверяем $q$-ветвление функции $U$ с коэффициентом $q^{-2}$.

Таким образом, согласно определению (16) и следствию 7 матрица-функция $V^{j}$ непрерьвна в $\bar{\Omega}$.

4. Обоснование асимптотики. Определим срезающую функцию $X^{h}$ по формуле

$$
X^{h}\left(x_{2}\right)=1-\chi\left(h^{-1} x_{2}\right)-\chi\left(h^{-1}\left(a_{2}-x_{2}\right)\right),
$$

где $\chi(t) \in C^{\infty}\left(\mathbb{R}^{1}\right)$ - эталонная "срезка", $\chi(t)=1$ при $|t|<1 / 3$ и $\chi(t)=0$ при $|t|>2 / 3$. Вспоминая анзацы (8) и (16), в качестве приближенного решения $\mathscr{U}^{h}$ задачи $(4),(5)$ берем сумму

$$
\mathscr{U}^{h}(x)=v(x)+h X^{h}(x)^{[0]}(h, x) D\left(\nabla_{x}\right) v(x),
$$


где $\mathscr{V}$ - матрица-функция, определенная в полоске $\Pi_{j}(h)$ по формуле

$$
\mathscr{V}\left(x, \eta^{(j)}, \zeta\right)=w(x, \zeta)+q^{-j} W\left(x, \eta^{(j)}, \zeta\right)+q^{-2 j} U\left(x, \eta^{(j)}, \zeta\right)
$$

Для облегчения преобразований примем обозначения из п. 2 , но напомним, что они истолковываются в соответствии с п. 3 . Кроме того, пользуемся обозначениями $D\left(\nabla_{x}\right)$ для полной производной и $D_{x}, D_{\xi}$ для частных производных (см. (9)), а срезающую функцию условно считаем зависящей от медленной переменной $x_{2}$.

Разность $\mathscr{R}^{h}=u^{h}-\mathscr{U}^{h}$ удовлетворяет уравнению

$$
\mathscr{L} \mathscr{R}^{h}=\mathscr{L} u^{h}-\mathscr{L} \mathscr{U}^{h}=f-\mathscr{L} \mathscr{U}^{h},
$$

а также условиям Дирихле и периодичности (5). В силу лемм 1,5 и определения срезки $X^{h}$ справедливо включение $\mathscr{R}^{h} \in \stackrel{\circ}{H}_{\mathrm{per}}^{1}(\Omega)$. Умножим уравнение $(33)$ скалярно на $\mathscr{R}^{h}$ и проинтегрируем по частям при учете условий (5). Ввиду неравенства Корна (6) имеем

$$
-\left(\mathscr{L} \mathscr{U}^{h}-f^{h}, \mathscr{R}^{h}\right)_{\Omega}=\left(A^{[0]} D\left(\nabla_{x}\right) \mathscr{R}^{h}, D\left(\nabla_{x}\right) \mathscr{R}^{h}\right)_{\Omega} \geqslant c\left\|\mathscr{R}^{h}, H^{1}(\Omega)\right\|^{2}
$$

Здесь и далее $(\cdot, \cdot)_{\Omega}$ - скалярное произведение в пространстве $L_{2}(\Omega)$.

Выполнив дифференцирование в левой части (34), получаем

$$
\begin{aligned}
\mathscr{L} \mathcal{U}^{h}-f^{h}= & -h^{-1}\left\{D_{\xi}^{\top} A^{[0]} D_{x} v+D_{\xi}^{\top} A^{[0]} D_{\xi} \mathscr{V}^{[0]} D_{x} v\right\} \\
& -\left\{D_{x}^{\top} A^{[0]} D_{x} v+D_{x}^{\top} A^{[0]} D_{\xi} \mathcal{V}^{[0]} D_{x} v+f^{h}\right\} \\
& +\left\{D\left(\nabla_{x}\right)^{\top} A^{[0]}\left(1-X^{h}\right) D_{\xi}{ }^{[0]} D_{x} v-h D\left(\nabla_{x}\right)^{\top} A^{[0]} D_{x} X^{h}{ }^{[0]} D_{x}\right\} .
\end{aligned}
$$

Интегрируя по частям, представим скалярное произведение слева в (34) как сумму выражений

$$
\begin{aligned}
h^{-1} I^{(-1)} & =h^{-1}\left(D_{\xi}^{\top} A^{[0]} D_{x} v+D_{\xi}^{\top} A^{[0]} D_{\xi} \mathscr{V}^{[0]} D_{x} v, \mathscr{R}\right)_{\Omega}, \\
I^{(0)} & =\left(D_{x}^{\top} A^{[0]} D_{x} v+D_{x}^{\top} A^{[0]} D_{\xi} \mathcal{V}^{[0]} D_{x} v+f^{h}, \mathscr{R}\right)_{\Omega}, \\
I_{X}^{(0)} & =\left(A^{[0]}\left(1-X^{h}\right) D_{\xi}{ }^{[0]} D_{x} v, D\left(\nabla_{x}\right) \mathscr{R}\right)_{\Omega}, \\
h^{1} I_{X}^{(1)} & =-h\left(A^{[0]} D_{x} X^{h_{y}}{ }^{[0]} D_{x} v, D\left(\nabla_{x}\right) \mathscr{R}\right)_{\Omega} .
\end{aligned}
$$

Оценим эти выражения поочередно, начиная с последнего. Имеем

$$
\begin{gathered}
h\left|I_{X}^{(1)}\right|+\left|I_{X}^{(0)}\right| \leqslant \\
\sup _{x \in \Omega}\left\{\left|\mathcal{V}^{[0]}\right|+\left|D_{x} \mathscr{V}^{[0]}\right|+\left|D_{\xi} \mathscr{V}^{[0]}\right|\right\}\left(\left\|\nabla_{x} v ; L_{2}\left(\omega^{h}\right)\right\|\right. \\
\left.+h\left\|\nabla_{x}^{2} v ; L_{2}(\Omega)\right\|\right)\left\|D\left(\nabla_{x}\right) \mathscr{R} ; L_{2}(\Omega)\right\|,
\end{gathered}
$$

где $\omega^{h}=\Omega \cap \operatorname{supp}\left(1-X^{h}\right)$ - объединение двух полосок, имеющих ширину $2 h / 3$ и примыкаюших к основаниям $\left(0, a_{1}\right) \times\{0\}$ и $\left(0, a_{1}\right) \times\left\{a_{2}\right\}$ прямоугольника $\Omega$. Предположим, что вьполнены условия леммы 5 . В силу неравенства

$$
\left\|\mathscr{Z} ; L_{2}\left(\omega^{h}\right)\right\| \leqslant c h^{1 / 2}\left\|\mathscr{Z} ; H^{1}(\Omega)\right\| \quad \forall \mathscr{Z} \in H^{1}(\Omega),
$$

(см. [10, лемма 1.2.4]) и неравенства (29) справедлива оценка

$$
\left\|\nabla_{x} v ; L_{2}\left(\omega^{h}\right)\right\|+h\left\|\nabla_{x}^{2} v ; L_{2}(\Omega)\right\| \leqslant c h^{1 / 2}\left\|\mathbf{f} ; H^{\varkappa}(\Omega)\right\| .
$$


По определению (32) матрищы-функции $\mathscr{V}$ выполняется соотношение $\mathscr{V}[0]=w^{[0]}+$ $W^{[1]}+U^{[2]}$. Таким образом, благодаря следствию 7 матрица-функция $\mathscr{V}^{[0]}$, а значит, и $D_{x} \mathscr{V}^{[0]}$ непрерывны в $\bar{\Omega}$. Для проверки непрерьвности производной $D_{\xi} \mathcal{V}^{[0]}$ в $\bar{\Omega}$ докажем, что скачок $D_{\xi} \mathcal{V}^{[0]}$ этой производной на линии $\Gamma_{j}$ равен нулю. Для скачка $D_{\xi} W^{[1]}$ согласно (17) получаем

$$
\begin{aligned}
& D_{\xi}\left(q^{-j-1} W\left(x, \eta^{(j+1)},(j+1) b_{2}+0\right)-q^{-j} W\left(x, \eta^{(j)}, j b_{2}+b_{2}-0\right)\right) \\
& =D_{1}\left(\partial_{\eta} W\left(x, q \eta^{(j)},+0\right)-\partial_{\eta} W\left(x, \eta^{(j)}, b_{2}-0\right)\right) \\
& \quad+D_{2}\left(q^{-j-1} \partial_{\zeta} W\left(x, q \eta^{(j)},+0\right)-q^{-j} \partial_{\zeta} W\left(x, \eta^{(j)}, b_{2}-0\right)\right)=0 .
\end{aligned}
$$

Последнее равенство выполняется потому, что у $W$ имеется $q$-ветвление с коэффициентом $q^{-1}$, а производная $\partial_{\eta} W$ является периодической с $q$-ветвлением матрицей-функцией. Аналогично проверяется, что матрицы-функции $D_{\xi} w^{[0]}$ и $D_{\xi} U^{[2]}$ непрерывны на $\Gamma_{j}$. Таким образом,

$$
\sup _{x \in \Omega}\left\{\left|\mathscr{V}^{[0]}\right|+\left|D_{x} \mathscr{V}^{[0]}\right|+\left|D_{\xi} \mathscr{V}^{[0]}\right|\right\} \leqslant c h^{0}
$$

и, следовательно, при учете (36) и (37) окончательно устанавливаем, что

$$
h\left|I_{X}^{(1)}\right|+\left|I_{X}^{(0)}\right| \leqslant c h^{1 / 2}\left\|\mathbf{f} ; H^{\varkappa}(\Omega)\right\|\left\|D\left(\nabla_{x}\right) \mathscr{R} ; L_{2}(\Omega)\right\|
$$

Подчеркнем, что поскольку при оценке величины $h\left|I_{X}^{(1)}\right|+\left|I_{X}^{(0)}\right|$ (а также далее при рассмотрении слагаемых $h\left|I^{(1)}\right|$ и $\left.\left|I^{(0)}\right|\right)$ используются интегральные нормы величин $\mathscr{R}^{h}$ и $D_{x} v$, для сомножителя $\mathscr{V}^{[0]}$ приходится применять поточечную оценку (37), обеспеченную указанным в следствии 7 свойством членов суммы (16).

Для обработки $I^{(0)}$ необходимо уточнить информацию о правой части $f^{h}=F^{[0]}$ системы (4): будем считать, что при некотором $\varkappa \in(0,1 / 2]$ справедливо включение

$$
F \in H^{\varkappa}\left(\Omega \rightarrow L_{\infty}(S)\right)^{n}
$$

где $H^{\varkappa}\left(\Omega \rightarrow L_{\infty}(S)\right)$ - пространство, полученное пополнением линеала $C_{\mathrm{per}}^{\infty}(\Omega \times S)$ функций, периодических относительно переменной $x_{1} \in\left(0, a_{1}\right]$, по норме

$$
\begin{aligned}
\left\|F ; H^{\varkappa}\left(\Omega \rightarrow L_{\infty}(S)\right)\right\|= & \left(\int_{\Omega} \sup _{y \in S}|F(x, y)|^{2} d x\right. \\
& \left.+\int_{\Omega} \int_{\Omega} \sup _{y \in S}|F(x, y)-F(\mathbf{x}, y)|^{2} \frac{d x d \mathbf{x}}{|x-\mathbf{x}|^{2(1+\varkappa)}}\right)^{1 / 2} .
\end{aligned}
$$

Если вектор-функция $F$ не зависит от $y$, то норма (39) совпадает с обычной нормой в пространстве Соболева-Слободецкого $H^{\varkappa}(\Omega)$ и не превосходит нормы в пространстве $H^{1}(\Omega)$. Подчеркнем, что при $\varkappa<1 / 2$ функция $F$ не сохраняет периодичность по $x_{1}$.

Понадобится также следующее утверждение, которое проверяется по схеме, аналогичной доказательству леммы 6.4.6 [10]. 
Лемма 8. Пусть $R \in \stackrel{\circ}{H}_{\text {per }}^{1}(\Omega), \mathscr{T} \in H^{\varkappa}\left(\Omega \rightarrow L_{\infty}(S)\right) u$

$$
\overline{\mathscr{T}}(x)=\left(\operatorname{mes}_{2} S\right)^{-1} \int_{S} \mathscr{T}(x, \eta, \zeta) d \eta d \zeta
$$

\section{Тогда выполняется неравенство}

$$
\left|\int_{\Omega}\left(\mathscr{T}^{[0]}\left(x, \eta^{(j)}, \zeta\right)-\overline{\mathscr{T}}(x)\right) R(x) d x\right| \leqslant c h^{\varkappa}\left\|\mathscr{T} ; H^{\varkappa}\left(\Omega \rightarrow L_{\infty}(S)\right)\right\|\left\|R ; H^{1}(\Omega)\right\| .
$$

ДокАЗАТЕЛЬСТВо. Для каждой ячейки $S_{j}^{p}(h)$ положим

$$
R_{j}^{p}=\left(\operatorname{mes}_{2} S_{j}^{p}(h)\right)^{-1} \int_{S_{j}^{p}(h)} R(x) d x .
$$

По неравенствам Пуанкаре и Коши-Буняковского имеем

$$
\left\|R-R_{j}^{p} ; L_{2}\left(S_{j}^{p}(h)\right)\right\|^{2} \leqslant c h^{2}\left\|\nabla R ; L_{2}\left(S_{j}^{p}(h)\right)\right\|^{2}, \quad\left|R_{j}^{p}\right|^{2} \leqslant c h^{-2} q^{-j}\left\|R ; L_{2}\left(S_{j}^{p}(h)\right)\right\|^{2} .
$$

Разбивая область на ячейки, получаем

$$
\begin{aligned}
& \int_{\Omega}\left(\mathscr{T}^{[0]}\left(x, \eta^{(j)}, \zeta\right)-\overline{\mathscr{T}}(x)\right) R(x) d x \\
& \leqslant \sum_{j=1}^{N_{2}} \sum_{p=1}^{N_{1}} q^{j}\left\{\int_{S_{j}^{p}(h)}\left|\mathscr{T}\left(x, h^{-1} q^{j} x_{1}, h^{-1} x_{2}\right)-\overline{\mathscr{T}}(x)\right|\left|R(x)-R_{j}^{p}\right| d x\right. \\
& \left.\quad+\left|R_{j}^{p}\right|\left|\int_{S_{j}^{p}(h)}\left(\mathscr{T}\left(x, h^{-1} q^{j} x_{1}, h^{-1} x_{2}\right)-\overline{\mathscr{T}}\right)(x) d x\right|\right\}=: \sum_{j=1}^{N_{2}} \sum_{p=1}^{N_{1}} q^{j}\left(I_{j}^{p}+J_{j}^{p}\right) .
\end{aligned}
$$

Согласно (40) при любом $\varepsilon>0$

$$
\begin{aligned}
I_{j}^{p} & \leqslant \operatorname{ch}\left(\left\|\mathscr{T} ; L_{2}\left(S_{j}^{p}(h)\right)\right\|+\left\|\overline{\mathscr{T}} ; L_{2}\left(S_{j}^{p}(h)\right)\right\|\right)\left\|\nabla R ; L_{2}\left(S_{j}^{p}(h)\right)\right\| \\
& \leqslant \operatorname{ch}\left(\varepsilon\left\|\mathscr{T} ; L_{2}\left(S_{j}^{p}(h) \rightarrow L_{\infty}(S)\right)\right\|^{2}+\varepsilon^{-1}\left\|\nabla R ; L_{2}\left(S_{j}^{p}(h)\right)\right\|\right) .
\end{aligned}
$$

Наконец, в силу (40) имеем

$$
\begin{aligned}
J_{j}^{p} \leqslant & c h^{-1} q^{-j / 2}\left\|R ; L_{2}\left(S_{j}^{p}(h)\right)\right\| \int_{S_{j}^{p}(h)}\left|\mathscr{T}\left(x, h^{-1} q^{j} x_{1}, h^{-1} x_{2}\right)-\overline{\mathscr{T}}(x)\right| d x \\
= & c h^{-1} q^{-j / 2}\left\|R ; L_{2}\left(S_{j}^{p}(h)\right)\right\|\left(\operatorname{mes}_{2} S_{j}^{p}(h)\right)^{-1} \int_{S_{j}^{p}(h)} \int_{S_{j}^{p}(h)} \mid \mathscr{T}\left(x, h^{-1} q^{j} x_{1}, h^{-1} x_{2}\right) \\
& \quad-\bar{T}(x)-\mathscr{T}\left(y, h^{-1} q^{j} x_{1}, h^{-1} x_{2}\right)+\overline{\mathscr{T}}(y) \mid d x d y \\
\leqslant & c h^{-1} q^{-j / 2}\left\|R ; L_{2}\left(S_{j}^{p}(h)\right)\right\| h^{1+\varkappa}\left(\int_{S_{j}^{p}(h)} \int_{S_{j}^{p}(h)} \mid \sup \left\{\mid \mathscr{T}^{[0]}(x, \eta)-\overline{\mathscr{T}}(x)\right.\right. \\
& \left.\left.\quad-\mathscr{T}^{[0]}(y, \eta)+\overline{\mathscr{T}}(y) \mid ; \eta \in S\right\}\left.\right|^{2}|x-y|^{-2-2 \varkappa} d x d y\right)^{1 / 2} \cdot
\end{aligned}
$$


Пояснение: в подынтегральноевыражение добавили слагаемое с нулевьм средним, а затем оценили по максимуму модуля. Подчеркнем, что $|x-y| \leqslant\left(h^{2} a_{2}^{2}+h^{2} q^{-2 j} a_{1}^{2}\right)^{1 / 2} \leqslant c h$ при $x, y \in S_{j}^{p}(h)$, и поэтому введение множителя $|x-y|^{-2-2 \varkappa}$ позволило написать $h^{1+\varkappa}$ перед скобкой. Итак,

$$
J_{j}^{p} \leqslant c h^{\varkappa} q^{-j / 2}\left(\varepsilon\left\|\mathscr{T}: H^{\varkappa}\left(\Omega \rightarrow L_{\infty}(S)\right)\right\|^{2}+\varepsilon^{-1}\left\|R ; L_{2}\left(S_{j}^{p}(h)\right)\right\|^{2}\right) .
$$

Осталось просуммировать неравенства (41) и (42) по всем ячейкам и воспользоваться простьм алгебраическим фактом: если $c_{k}^{2} \leqslant \varepsilon a_{k}^{2}+\varepsilon^{-1} b_{k}^{2}$ при любом $\varepsilon>0$, то

$$
\sum_{k=1}^{K} c_{k}^{2} \leqslant 2\left(\sum_{k=1}^{K} a_{k}^{2}\right)^{1 / 2}\left(\sum_{k=1}^{K} b_{k}^{2}\right)^{1 / 2}
$$

При $a_{1}=\cdots=a_{K}=0$ неравенство очевидно, поскольку $c_{1}=\cdots=c_{K}=0$ ввиду произвольности $\varepsilon$; в противном случае выберем $\varepsilon=\left(\sum a_{k}^{2}\right)^{-1 / 2}\left(\sum b_{k}^{2}\right)^{1 / 2}$.

Учитывая уравнение $\left(7_{1}\right)$, представим слагаемое $I^{(0)}$ из $(35)$ как сумму двух интегралов

$$
\begin{aligned}
I^{(0)}= & \int_{\Omega}\left\{F^{[0]}-\bar{F}+D_{x}\left(\mathfrak{A}^{[0]}-\overline{\mathfrak{A}}\right) D_{x} v\right\} \mathscr{R}^{h} d x \\
& +\int_{\Omega}\left(D_{x}\left(\mathfrak{W}^{[1]}+\mathfrak{U}^{[2]}\right) D_{x} v\right) \mathscr{R}^{h} d x=: I_{1}^{(0)}+I_{2}^{(0)},
\end{aligned}
$$

где

$$
\mathfrak{A}=A+A\left(D_{\zeta} w+D_{\eta} W\right), \quad \mathfrak{W}=A\left(D_{\zeta} W+D_{\eta} U\right), \quad \mathfrak{U}=A D_{\zeta} U .
$$

Поскольку средние по ячейке от разностей, помещенных в фигурные скобки в (43), равны нулю, для оценки интеграла $I_{1}^{(0)}$ применяем лемму 8. В результате при учете неравенства (29) для $v$ обнаруживаем, что

$$
\begin{aligned}
\left|I_{1}^{(0)}\right| & \leqslant \operatorname{ch}^{\varkappa}\left\|F ; H^{\varkappa}\left(\Omega \rightarrow L_{\infty}(S)\right)\right\|\left\|\mathscr{R}^{h} ; H^{1}(\Omega)\right\|\left\|v ; H^{\varkappa+2}(\Omega)\right\| \\
& \leqslant \operatorname{ch}^{\varkappa}\left\|F ; H^{\varkappa}\left(\Omega \rightarrow L_{\infty}(S)\right)\right\|\left\|\mathscr{R}^{h} ; H^{1}(\Omega)\right\| .
\end{aligned}
$$

Рассмотрим интеграл $I_{2}^{(0)}$. Поскольку

$$
(j-1) h b_{2}<x_{2} \leqslant j h b_{2} \quad \text { при } \quad x \in \Pi_{j}(h),
$$

и, следовательно, $q^{-j} x_{2} \leqslant c h$, находим, что в силу формул (3) и (44) для выражений $\mathfrak{W}^{[1]}$ и $\mathfrak{U}^{[2]}$ справедливы неравенства

$$
s_{p}:=\sup _{x \in \Omega}\left|x_{2} D_{x}^{p}\left(\mathfrak{W}^{[1]}+\mathfrak{U}^{[2]}\right)\right| \leqslant c_{p} h, \quad p=0,1 .
$$

Теперь при помощи неравенства Харди

$$
\left\|x_{2}^{-1} \mathscr{Z} ; L_{2}(\Omega)\right\| \leqslant c\left\|\nabla_{x} \mathscr{Z} ; L_{2}(\Omega)\right\| \quad \forall \mathscr{Z} \in \stackrel{\circ}{H}_{\mathrm{per}}^{1}(\Omega)
$$


выводим такую оценку для $I_{2}^{(0)}$ :

$$
\begin{aligned}
\left|I_{2}^{(0)}\right| & \leqslant c \sum_{p=0}^{1} s_{p}\left\|v ; H^{2-p}(\Omega)\right\| \cdot\left\|x_{2}^{-1} \mathscr{R}^{h} ; L_{2}(\Omega)\right\| \\
& \leqslant c h^{1}\left\|F ; H^{\varkappa}\left(\Omega \rightarrow L_{\infty}(S)\right)\right\| \cdot\left\|\mathscr{R}^{h} ; H^{1}(\Omega)\right\| .
\end{aligned}
$$

Осталось обработать выражение $h^{-1} I^{(-1)}$. Для этого наряду с (46) понадобится еще одно неравенство

$$
\left\|D\left(\partial_{x}\right) v ; C(\bar{\Omega})\right\| \leqslant c\left\|v ; H^{\varkappa+2}(\Omega)\right\|
$$

вытекающее из теоремы вложения $H^{\varkappa+2} \subset C^{1}$.

По построению сумма $\mathscr{V}$ удовлетворяет уравнению $(10)$ в полосе $\Pi_{j}(h)$ с точностью $O\left(q^{-j}\right)$, т.е. вьполняется неравенство

$$
\sup _{x \in \Pi_{j}(h)}\left|D_{\xi}^{\top} A D_{\xi} \mathcal{V}^{j}+D_{\xi}^{\top} A\right| \leqslant c q^{-j} .
$$

Малость левой части (48) обеспечена лишь при больших $j$; однако для оценки величины $h^{-1}\left|I^{(-1)}\right|$ достаточно применить соотношение (48). Учитывая (46) и (47), при любом $\varepsilon>0$ вьводим цепочку неравенств

$$
\begin{aligned}
h^{-2}\left|I^{(-1)}\right| & \leqslant c\left(\varepsilon^{-1}\left\|x_{2}^{-1} \mathscr{R}^{h} ; L_{2}(\Omega)\right\|^{2}+c \varepsilon h^{-2} \sum_{j=1}^{N_{2}-1} \int_{j h}^{(j+1) h} x_{2}^{2} d x\right)^{1 / 2}\left\|D\left(\partial_{x}\right) v ; C(\bar{\Omega})\right\| \\
& \leqslant c h^{1 / 2}\left\|F ; H^{\varkappa}\left(\Omega \rightarrow L_{\infty}(S)\right)\right\| \cdot\left\|\mathscr{R}^{h} ; H^{1}(\Omega)\right\| .
\end{aligned}
$$

В последнем преобразовании были использованы оценки (29) и (43). Собирая полученные оценки величин (35) в левой части (34), приходим к следующему утверждению.

Теорема 9. Если матрица D, матрица-функиия $A$ и вектор-функиия $f^{h}$ удовлетворяют указанным выше предположениям, то выполняется оценка

$$
\left\|u^{h}-\mathscr{U}^{h} ; L_{2}(\Omega)\right\|+\left\|\nabla_{x}\left\{u^{h}-\mathscr{U}^{h}\right\} ; L_{2}(\Omega)\right\| \leqslant \operatorname{ch}^{\varkappa}\left\|F ; H^{\varkappa}\left(\Omega \rightarrow L_{\infty}(S)\right)\right\|,
$$

с постоянной с, не зависящей от функиии $F$ и параметра $h \in\left(0, h_{0}\right]$.

Удалим из глобального приближения “лишние" слагаемые, введенные для технических целей, и получим окончательные асимптотические формулы для решения $u^{h} \in$ $\stackrel{\circ}{H}_{\mathrm{per}}^{1}(\Omega)^{n}$ задачи (4) и его градиента.

ТЕОРема 10. При прежнних условиях справедливо неравенство

$$
\left\|u^{h}-v ; L_{2}(\Omega)\right\|+\left\|\nabla_{x} u^{h}-\nabla_{x} v-\mathfrak{w}^{[0]} D\left(\nabla_{x}\right) v ; L_{2}(\Omega)\right\| \leqslant c h^{\varkappa}\left\|F ; H^{\varkappa}\left(\Omega \rightarrow L_{\infty}(S)\right)\right\|,
$$

в котором $\mathfrak{w}=\left\{\left(\partial_{\eta}, 0\right) W+\left(0, \partial_{\zeta}\right) w\right\}$, а постоянная с не зависит от функиии $F u$ параметра $h \in\left(0, h_{0}\right]$. 


\section{СПИСОК ЦИТИРОВАННОЙ ЛИТЕРАТУРЫ}

[1] Nečas J. Les méthodes directes en théorie des équations elliptiques. Paris: Masson, 1967.

[2] Назаров С. А. Полиномиальное свойство самосопряженных эллиптических краевых задач и алгебраическое описание их атрибутов // УМН. 1999. Т. 54. № 5. С. 77-142.

[3] Назаров С. А. Самосопряженные эллиптические краевые задачи. Полиномиальное свойство и формально положительные операторы // Проблемы матем. анализа. Вып. 16. СПб.: Изд-во СПбГУ, 1997. С. 167-192.

[4] Kozlov S. M. Harmonization and homogenization on fractals // Comm. Math. Phys. 1993. V. 153. P. 339-357.

[5] ЖКиков В.В. Связность и усреднение. Примеры фрактальной проводимости // Матем. сб. 1996. T. 186. № 8. C. 3-40.

[6] Бахвалов Н. С., Панасенко Г.А. Осреднение процессов в периодических средах. М.: Наука, 1984.

[7] Санчес-Паленсия Э. Неоднородные среды и теория колебаний. М.: Мир, 1984.

[8] Олейник О. А. Иосифьян Г. А., Шамаев А. С. Математическиезадачи теории сильно неоднородных упругих сред. М.: Изд-во МГУ, 1990.

[9] Mazja W. G., Nazarov S. A., Plamenewski B. A. Asymptotische Theorie elliptischer Randwertaufgaben in singulär gestörten Gebieten. V. 2. Berlin: Akademie-Verlag, 1991.

[10] Назаров С. А. Асимптотический анализ тонких пластин и стержней. Т. 1. Понижение размерности и интеграљьые оценки. Новосибирск: Научная книга, 2002.

[11] Назаров С. А. Общая схема осреднения самосопряженных эллиптических систем в многомерных областях, в том числе тонких // Алгебра и анализ. 1995. Т. 7. № 5. С. 1-92.

[12] Agmon S., Douglis A., Nirenberg L. Estimates near the boundary for solutions of elliptic differential equations satisfying general boundary conditions. 2 // Comm. Pure Appl. Math. 1964. V. 17. № 1. P. 35-92.

Институт проблем машиноведения РАН, г. Санкт-Петербург

Поступило E-mail: serna@snark.ipme.ru, andr@AS2607.spb.edu 